\title{
Niching Genetic Algorithms for Optimization in Electromagnetics II. Shape Optimization of Electrodes using the CSM
}

\author{
B. Sareni, L. Krähenbühl, D. Muller \\ CEGELY - UPRESA CNRS 5005 - Ecole Centrale de Lyon \\ BP 163 - 69131 Ecully Cedex - France.
}

\begin{abstract}
In this paper, we present a new approach for automatic design of electrodes. The investigated method consists in identifying an optimal shape from an optimal equipotential resulting from a system of point charges. The electric field and potential are computed using the point charge simulation method. Niching genetic algorithms and constrained optimization techniques are applied to the electrode benchmark in order to find multiple optimal profiles.
\end{abstract}

Index terms-Genetic algorithms, niching, shape optimization, constrained optimization, penalty techniques, electrodes, charge simulation method.

\section{INTRODUCTION}

In [1], we have outlined the interest of Niching Genetic Algorithms for optimization in Electromagnetics. Niching methods extend Genetic Algorithms (GAs) by promoting the formation of stable subpopulations in the neighborhood of local and global optima. In this paper, we present a process for automatic design of electrodes using the Charge Simulation Method (CSM) [2][3] coupled with niching and constrained optimization techniques. Our approach is radically different from those developed elsewhere. In effect, shape optimal design is generally carried out by directly parametrizing the shape of devices [3][4]. The proposed method consists in identifying an optimal shape from an optimal equipotential line resulting from a system of point charges. The study explores the efficiency of many niching GAs when applied to the proposed electrode benchmark : in this paper, the technological problems will not be discussed.

\section{ELECTRODES DESTGN}

\section{A. Principle}

We consider a 2D-electrode device as displayed in Fig. 1. The electrode shape must satisfy a given template (valid area) defined by an internal and an external boundary. For reason of simplicity (remember that we only want to determine an optimal electrode shape), we consider a mathematically equivalent problem. Consequently, we use normalized rather than physical dimensions for the potential, the field, the charges and the lengths. The electric potential results from four point charges lying on the $y$-axis at an ordinate between

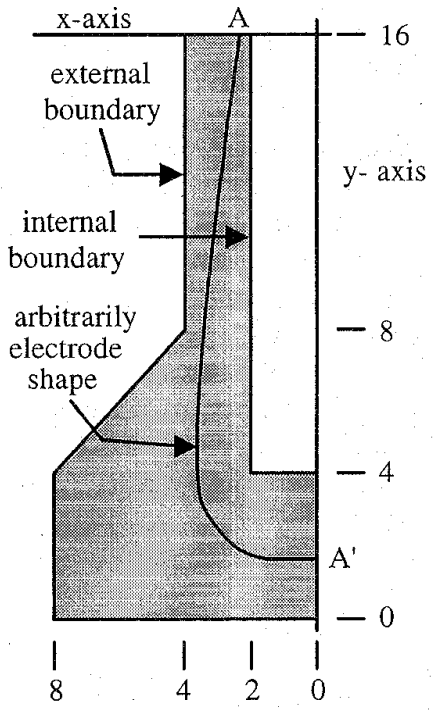

Fig. 1. Electrode template

4.5 and 16 . Each charge can take any value bounded by $q_{\min }$ and $q_{\max }$.

The system is considered to be symmetrical in relation to the $x$-axis and $y$-axis. Therefore, we must take into account four identical charges with symmetrical coordinates in relation to the $\mathrm{x}$-axis for the computation of the electric potential. We can express it at one point of coordinates $x$ and $y$ as follows,

$$
V(x, y)=-\frac{1}{2 \pi \varepsilon} \sum_{i=1}^{8} q_{i} \ln r_{i}
$$

where $q_{i}$ denotes the value of the charge $i$, $r_{i}=\sqrt{\left(x_{i}-x\right)^{2}+\left(y_{i}-y\right)^{2}}$ is the distance between that point and the corresponding charge. In the following, we simplify the analysis by neglecting the constant factor $-1 / 2 \pi \varepsilon$ in the computation of the potential and field values

Finding an optimal electrode shape consists of determining the equipotential of value $V_{o b j}$, being between the internal and external boundaries and with minimum electric field on it.

To fulfill the geometric constraints (electrode surface between the internal and external boundaries), the following inequations must be satisfied:

$$
V_{\max }^{\text {int }} \leq V_{o b j} \leq V_{\min }^{e x t}
$$


where $V_{\min }^{\text {ext }}$ and $V_{\max }^{\text {int }}$ represent the minimum potential value on the external boundary and the maximum potential value on the internal boundary respectively. It should be noted that a dual relation which also leads to a fulfillment of the constraints can be obtained by inverting the indexes ext and int.

The minimum and maximum possible values for each charge were set to $q_{\min }=-1$ and $q_{\max }=1$ respectively. Following this way, we search for an optimal equipotential (i.e. with minimum field on it) having for value $V_{o b j}=1$.

\section{B. Shape optimization of the electrode}

The optimization process that we employ can be described as follows:

1) Parameters optimization: Niching GAs optimize the set of parameters at each generation i.e. the characteristics of the charges (position and value).

2) Computation of the potential on the template boundaries: Before computing the objective function, namely the maximum electric field on the equipotential $V_{o b j}=1$, we must verify if geometric constraints are fulfilled. For that purpose, the internal and external boundaries are discretized into 15 and 25 points respectively. The potential is computed at each point and the extremum values of the potential on the boundaries are returned.

3) Constraints fulfillment: First investigations were made by taking two constraints relative to (2). This procedure was not efficient (see section III for more details). Therefore, we choose to use a normalization process to increase the feasible domain and reduce the number of constraints. In effect, we remark that a sufficient condition to have an equipotential of any value $\tilde{V}_{o b j}$ inside the template is simply:

$$
V_{\max }^{\text {int }} \leq V_{\min }^{e x t}
$$

4) Potential normalization: When (2) is reduced to (3), we obtain the equivalent charge values giving a corresponding equipotential of $V_{o b j}=1$ by multiplying the intensity of all charges by the term $1 / \tilde{V}_{o b j}$ where $\tilde{V}_{o b j}$ is a value lying in the range $V_{\max }^{\mathrm{int}} \leq \widetilde{V}_{o b j} \leq V_{\min }^{e x t}$. All of the possible values for $\tilde{V}_{o b j}$ can be defined by (4),

$$
\tilde{V}_{o b j}=V_{\text {rax }}^{\mathrm{int}}+a\left(V_{\min }^{e x t}-V_{\max }^{\mathrm{int}}\right)
$$

where $a \in[0,1]$ is called the normalization factor. We explore three different normalization techniques to set the normalization factor:

- The normalization factor is taken as an additional parameter and is optimized by the niching GA (GAnormalization).
- The normalization factor is independently optimized by a one-dimensional optimization method (optimized normalization). We apply the golden section method [6].

- The normalization factor is set to 1 (external normalization). This procedure is based on the assumption that optimal profiles must touch the external boundary of the template to present a minimal electric field on their surface.

5) Equipotential scanning: One point of the equipotential $\tilde{V}_{o b j}$ is detected along the $\mathrm{x}$-axis from the point of coordinates $(3,16)$ using the Newton-Raphson method [6]. The other points are sequentially found with the following HillClimbing procedure. The first point identified is placed at the center of a grid which can move in eight directions of the space according to two different pattern moves (diagonal and lateral moves). Fig. 2 presents two examples of pattern moves.

From a gradient corresponding to the last direction of the move (initially we impose the negative direction along the $y$ axis) the potential is computed at a limited number of points among the eight possible. We limit this number according to the gradient to five points for a diagonal move and three points for a lateral move (see Fig. 2). This was necessary to prevent reexploring points identified before. Following this way, the point that yields a minimum slope of potential with the center of the grid is retained as the new center. The scanning is stopped when the center of the grid intercepts the y-axis.

6) Maximum electric field: The electric field value is computed at each point of the equipotential detected by the previous procedure. The maximum value is returned and normalized by the term $1 / \tilde{V}_{o b j}$ according to the normalization factor defined in (4). The fitness function is defined as follows to express the problem into a form of maximization for the niching GAs,

$$
f_{G A}=1 /\left(1+E_{\max }\right)
$$

where $E_{\max }$ is the maximum normalized electric field value on the equipotential.

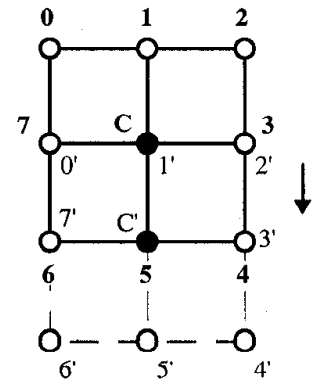

(a)

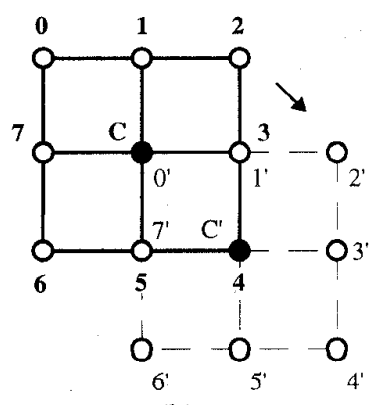

(b)
Fig. 2. Equipotential scanning (a) lateral move (b) diagonal move 


\section{7) Penalization process}

When the constraints are violated, we cannot compute the objective function since it has no meaning to evaluate the electric field on an equipotential lying outside the template. On the other hand, the equipotential corresponding to an unfeasible solution can take any shape outside the template, making its identification more difficult. For this reason, there is no possible link between the feasible and unfeasible domains. We investigate two penalty techniques to compute the fitness function for unfeasible solutions:

- We reject unfeasible individuals from the population by resetting their fitness (death penalty technique). This procedure is one of the most popular in evolutionary computing but it suffers from significant drawbacks. In effect, if no feasible solution is found in the initial population, there is no way to improve individuals and help them to reach the feasible domain

- Individuals are penalized by a quantity almost proportional to the violation of the constraints (exterior penalty technique). We use the following penalty term for the fitness function:

$$
f_{G A}=1 /\left(1+w_{1}+w_{2} \sum_{i=1}^{m} g_{i}\right)
$$

where $w_{1}$ and $w_{2}$ are fixed penalty parameters, $g_{i}$ represents a violated constraint, $m$ being the number of violated constraints. $w_{1}$ must be sufficiently large to prevent convergence on the external boundary of the feasible domain but not too high to avoid great discontinuity between feasible and unfeasible domains. This value has to be close to the worst feasible solution to ensure sufficient penalization.

\section{RESULTS}

Four niching methods reviewed in [1][7] were alternatively applied to solve the electrode benchmark. The population size of the GAs was $N=100$, the number of generations being $t_{\max }=200$. All parameters were coded in a 30 bit number with standard binary encoding. The crossover probability and the mutation rate were set to 1.0 and 0.001 respectively. Four runs were made with random populations to take into account the stochastic nature of the GAs. An average statistic was taken from the final population for the performance criteria: number of optimal profiles, best profile, and normalization factor.

\section{A. Niching GAs compared.}

Table I shows statistics obtained for the studied GAs with GA-normalization and death penalty method. A standard elitist GA converged to a single solution of the space but the four successive runs converged to four different solutions of quasi identical fitness reflecting the multimodality of the landscape (or the presence of a large, relatively flat area in it). Sharing was unable to find any optimal solutions resulting from a lack of elitism inherent to this method and probably an inappropriate niche radius and population size - sharing generally requires high population sizes to reach equilibrium [6]. The objective function seems to be a large plateau with a low gradient and one should probably improve the results by using fitness scaling to increase differentiation of optimal solutions. Clearing was more robust than sharing finding an important number of closed optimal solutions with a small niche radius $\left(\sigma_{s}=0.05\right)$ and a few (but more distinct) ones with higher niche radii $\left(\sigma_{s}=0.2\right.$ or $\left.\sigma_{s}=0.4\right)$. Restricted Tournament Selection performed well by maintaining a higher number of distinct optimal profiles. Deterministic Crowding only detected an average of 2.25 optima in four runs.

TABLE I

COMPARISON OF DIFFERENT NICHING GENETIC ALGORITHMS ON THE ELECTRODE BENCHMARK

\begin{tabular}{ccc}
\hline GA optimization & $\begin{array}{c}\text { Number of } \\
\text { optimal profiles } \\
(\text { Emax<0.06) }\end{array}$ & $\begin{array}{c}\text { Best } \\
\text { solution } \\
\text { (Emax) }\end{array}$ \\
\hline elitist GA(no niching) & 1 & 0.0564 \\
Sharing $\left(\sigma_{\mathrm{s}}=0.05\right)$ & 0 & 0.0672 \\
Sharing $\left(\sigma_{\mathrm{s}}=0.2\right)$ & 0 & 0.0668 \\
Sharing $\left(\sigma_{\mathrm{s}}=0.4\right)$ & 0 & 0.0746 \\
Clearing $\left(\sigma_{\mathrm{s}}=0.05\right)$ & 60.50 & 0.0561 \\
Clearing $\left(\sigma_{\mathrm{s}}=0.2\right)$ & 16.25 & 0.0568 \\
Clearing $\left(\sigma_{\mathrm{s}}=0.4\right)$ & 7.50 & 0.0559 \\
DC & 2.25 & 0.0569 \\
RTS & 18.50 & 0.0567 \\
\hline
\end{tabular}

\section{B. Normalization methods compared.}

We present in Table II statistics obtained for Restricted Tournament Selection coupled with death penalty method and the normalization techniques introduced in section II.

TABLE II

COMPARISON OF THE NORMALIZATION TECHNIQUES FOR RTS

\begin{tabular}{|c|c|c|c|}
\hline Normalization & $\begin{array}{c}\text { Average normalization } \\
\text { factor for optimal } \\
\text { profiles }\end{array}$ & $\begin{array}{c}\text { Number of } \\
\text { optimal profiles } \\
(\text { Emax }<0.06)\end{array}$ & $\begin{array}{c}\text { Best } \\
\text { solution } \\
(\text { Emax })\end{array}$ \\
\hline$G A$ & 0.663 & 18.50 & 0.0567 \\
\hline optimized $^{1}$ & 0.994 & 9.00 & 0.0563 \\
\hline external & 1 (fixed) & 11.50 & 0.0561 \\
\hline
\end{tabular}

Results are quite similar for the 3 schemes investigated. External normalization was the most efficient method since optimal solutions are located on the boundary of the domain corresponding to a normalization factor of value 1.0. This is proved by optimized normalization which always leads to a normalization factor of 0.994 for optimal solutions. This last method is the most general and theoretically the best of the three ones. However, it requires more CPU time because of one equipotential scanning per division in the golden section method. GA normalization yielded a higher number of optimal profiles by maintaining solutions with normalization factor of any value. Nevertheless, these solutions were worse

\footnotetext{
${ }^{1}$ For optimized normalization, the normalization factor was determined after 9 iterations of the golden section method.
} 
than those found by the previous methods. We point out that without normalization (i.e. when using fixed constraints relative to (2)) and death penalty method no feasible solution was found in 20000 fitness function evaluations for all GAs. In fact, 6819500 fitness function evaluations were necessary to evaluate 10 feasible solutions with random search.

\section{Penalty methods compared.}

Table III shows statistics obtained for different niching GAs with external normalization. Exterior penalty method was computed using $w_{1}=0.1$ (which corresponds to the worst value of the objective function in the feasible domain found with random search) and $w_{2}=100$.

TABLE III

COMPARISON OF THE PENALTY TECHNIQUES FoR DIFFERENT NICHING GAS

\begin{tabular}{ccccc}
\hline $\begin{array}{c}\text { Optimization } \\
\text { scheme }\end{array}$ & $\begin{array}{c}\text { Penalty } \\
\text { technque }\end{array}$ & $\begin{array}{c}\text { Exploration } \\
\text { rate }^{2}\end{array}$ & $\begin{array}{c}\text { Number of } \\
\text { optimal profiles } \\
(\text { Emax<0.06) }\end{array}$ & $\begin{array}{c}\text { Best } \\
\text { solution } \\
\text { (Emax) }\end{array}$ \\
\hline RTS & death & $8.20 \%$ & 11.50 & 0.0561 \\
RTS & exterior & $19.26 \%$ & 33.75 & 0.0561 \\
DC & death & $2.97 \%$ & 2.50 & 0.0571 \\
DC & exterior & $19.43 \%$ & 17.50 & 0.0563 \\
Clearing $\left(\sigma_{s}=0.2\right)$ & death & $64.96 \%$ & 17.00 & 0.0564 \\
Clearing $\left(\sigma_{s}=0.2\right)$ & exterior & $64.46 \%$ & 14.00 & 0.0563 \\
\hline
\end{tabular}

For all GAs, only a few feasible solutions were evaluated in the first generations. With death penalty method, crowding methods such as Deterministic Crowding or Restricted Tournament Selection were unable to improve the major part of the population located outside the feasible domain yielding a small exploration rate. Clearing rapidly converged and stabilized its population in the feasible domain due to the selection operator similar to that used in standard GAs. This explains the high exploration rate noted for that GA. The Exterior penalty method considerably improved the exploration rate and the quality of results for crowding schemes. It should be also noted that the exploration rate of a random search is extremely poor (about $0.8 \%$ ) whereas that of a standard GA with death penalty method is about $93 \%$ (remind that a standard GA rapidly converges to a single region of the feasible domain). We conclude this section by presenting in Fig. 3 examples of optimal profiles found and associated electric field stress on their contour in Fig. 4.

\section{CONCLUSION}

This paper describes an original approach for automatic design of electrode shapes. The procedure, based on the CSM coupled with niching $\mathrm{GAs}$, allows to find multiple optimal profiles with reduction of the maximum field stress. The paper also investigates normalization schemes and penalty methods to improve the efficiency of niching GAs. In the future, we will extend our method to three dimensional systems with more complex templates and charges with

${ }^{2}$ We define the exploration rate as the ratio of the number of computed feasible solutions to the total rumber of fitness function evaluations.

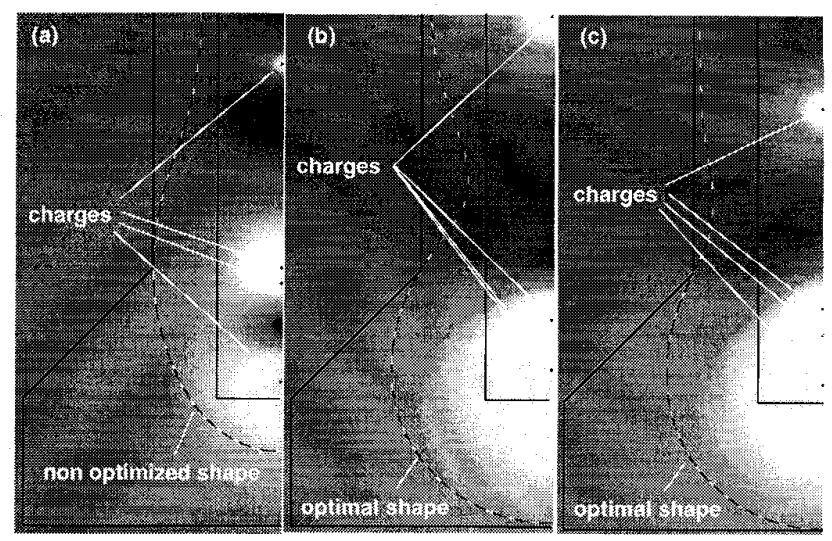

Fig. 3. Examples of profiles with the corresponding electric field distribution

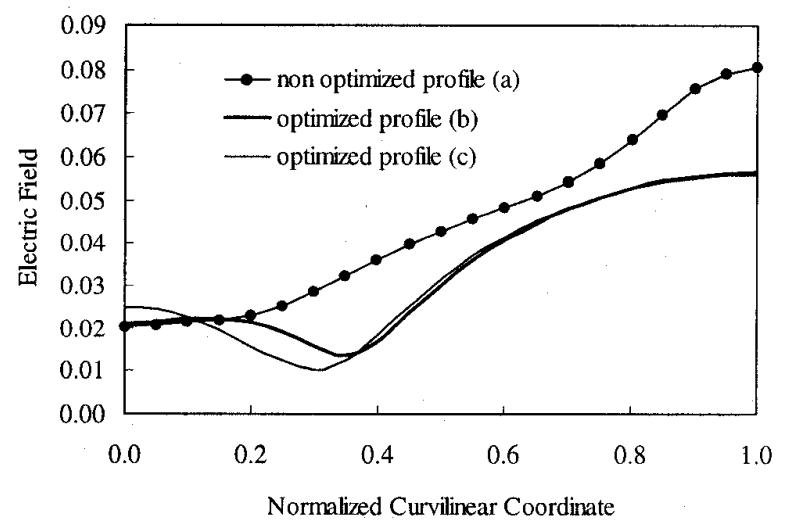

Fig. 4. Electric field stress on the countours.

additional degrees of freedom. A comparison of the efficiency of our approach will be carried out with other traditional shape optimization methods.

\section{REFERENCES}

[1] B. Sareni, L. Krähenbühl, A. Nicolas, "Niching Genetic Algorithms for Optimization in Electromagnetics I. Fundamentals," Int. Conf. Compumag, Rio, Brazil, nov. 1997.

[2] H. Singer, H. Steinbigler, P. Weiss, "A charge simulation method for the calculation of high voltage fields," IEEE Trans. Power Apparatus and Systems, vol. 93, No 5, pp. 1660-1667, 1974.

[3] P.K. Mukherjee, C. Trinitis, H. Steinbigler, "Optimization of HV Electrode Systems by Neural Networks using a New Learning Method," IEEE Trans. on D.E.I., vol. 3, No 6, pp. 737-742, 1996.

[4] J.A. Vasconcelos, L. Krähenbühl, L. Nicolas, A. Nicolas, "Design Optimization in Electrostatic Field Analysis Using the BEM and the Augmented Lagrangian Method," IEEE Trans. on Magn., vol. 30, No 5, pp. 3443-3446, 1994.

[5] Z. Michalewicz, D. Dasgupta, R. Leriche, M. Schoenauer, "Evolutionary AIgorithms for Constrained Engineering Problems," Computer and Industrial Engineering Journal, vol. 30, No 2, 1996.

[6] H.P. Schwefel, Evolution and Optimum Seeking, Sixth-Generation Computer Technology Series, Wiley, New York, 1995.

[7] S.W. Mahfoud, Niching Methods for Genetic Algorithms. IlliGAl Report 95001, University of llinois at Urbana Champaign, I.G.A. Lab. 1995. Available by ftp://gal4.ge.uiuc.edu/pub/papers/ 